\title{
SIBERIAN SNAKE EXPERIMENTS AT THE IUCF COOLER RING*
}

\author{
$\underline{\text { V.A. Anferov }}^{\dagger}$, B.B. Blinov, D.A. Crandell, Ya.S. Derbenev, T. Kageya, S.V. Koutin, A.D. Krisch, \\ R.A. Phelps, W. Lorenzon, L.G. Ratner, D.W. Sivers, K.V. Sourkont, V.K. Wong, S.S. Youssof, \\ Univ. of Michigan, Ann Arbor, MI \\ C.M. Chu, S.Y. Lee, T. Rinckel, P. Schwandt, F. Sperisen, B. von Przewoski, IUCF, Bloomington, IN \\ V.N. Grishin, P.A. Semenov, IHEP, Protvino, Russia \\ H. Sato, HEARO, Tsukuba, Japan
}

\section{Abstract}

Recent polarized proton beam experiments in the IUCF Cooler Ring found an evidence for a second-order snake depolarizing resonance, when the vertical betatron tune was inadvertently set near a quarter-integer. We have also studied the possibility of spin-flipping the beam polarization in the presence of a full Siberian snake using an rf solenoid. By varying the rf solenoid's ramp time and frequency range, we reached a spin-flip efficiency of about $97 \%$.

\section{SECOND-ORDER SNAKE DEPOLARIZING RESONANCE}

One must overcome many spin depolarizing resonances to accelerate a polarized proton beam to high energy. Earlier IUCF experiments [1] suggest that the Siberian snake technique [2] could universally overcome all intrinsic and imperfection depolarizing resonances even at high energy. However, another type of depolarizing resonances called "snake" resonances could occur at certain values of the vertical betatron tune even in the presence of a full Siberian snake in a ring. Using a $104 \mathrm{MeV}$ stored polarized proton beam and a full Siberian snake, we recently found evidence for a second-order "snake" depolarizing resonance.

In any circular accelerator or storage ring, each proton's spin precesses around the vertical fields of the ring's dipole magnets. The spin tune $\nu_{s}$, which is the number of spin precessions during one turn around the ring, is proportional to the proton's energy

$$
\nu_{s}=G \gamma
$$

where $\gamma$ is the Lorentz energy factor and $G=1.792847$ is the proton's anomalous magnetic moment. The beam can be depolarized when the spin precession frequency is synchronized with some horizontal magnetic field, which can be caused either by the ring's imperfections or by the vertical betatron oscillations. These spin perturbations are called imperfection and intrinsic depolarizing resonances, respectively; they occur when the spin tune is equal to an integer or to a harmonic of the betatron tune $\nu_{y}$ :

$$
\nu_{s}=G \gamma=n \pm k \nu_{y}
$$

* Supported by grants from the U.S. Department of Energy and National Science Foundation.

†Email: anferov@umich.edu where $n$ and $k$ are integers.

A full Siberian snake makes the spin tune energy independent at a half-integer; thus, it should overcome all imperfection and first-order intrinsic depolarizing resonances. However, in a ring with its betatron tune near a quarter integer, the snake can cause a second-order depolarizing resonance whenever

$$
\nu_{s}=n \pm 2 \nu_{y}
$$

where $n$ is an integer. Such a snake resonance [3] could depolarize a high-energy polarized beam.

We recently studied a second-order snake resonance using a 104.1 MeV polarized proton beam with a full Siberian snake in the IUCF Cooler Ring. The experimental apparatus, as shown in Fig. 1, included the snake's superconducting solenoid and eight correction quadrupoles, the polarimeter, the rf dipole, and the rf solenoid. The electron cooling section consists of the main solenoid, two toroidal magnets, two correction solenoids (CSA and CSB), and a set of vertical steerers to compensate for the orbit perturbation in the toroidal magnets.

It was shown earlier [4] that the electron cooling section produces an additional spin rotation which shifts the spin tune even in the presence of a full Siberian snake. By varying the current in the correction solenoids (CSA and CSB)

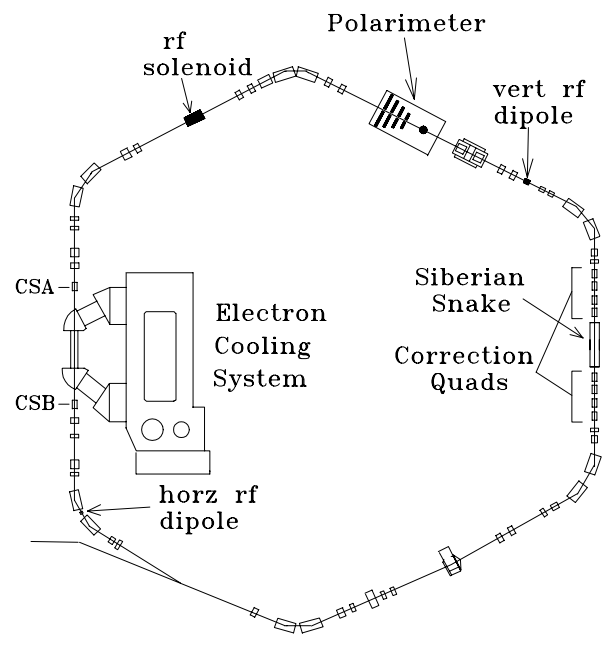

Figure 1: The IUCF Cooler Ring with the Siberian snake, the rf solenoid, the rf dipole, the polarimeter, and the CSA/CSB correction solenoids. 


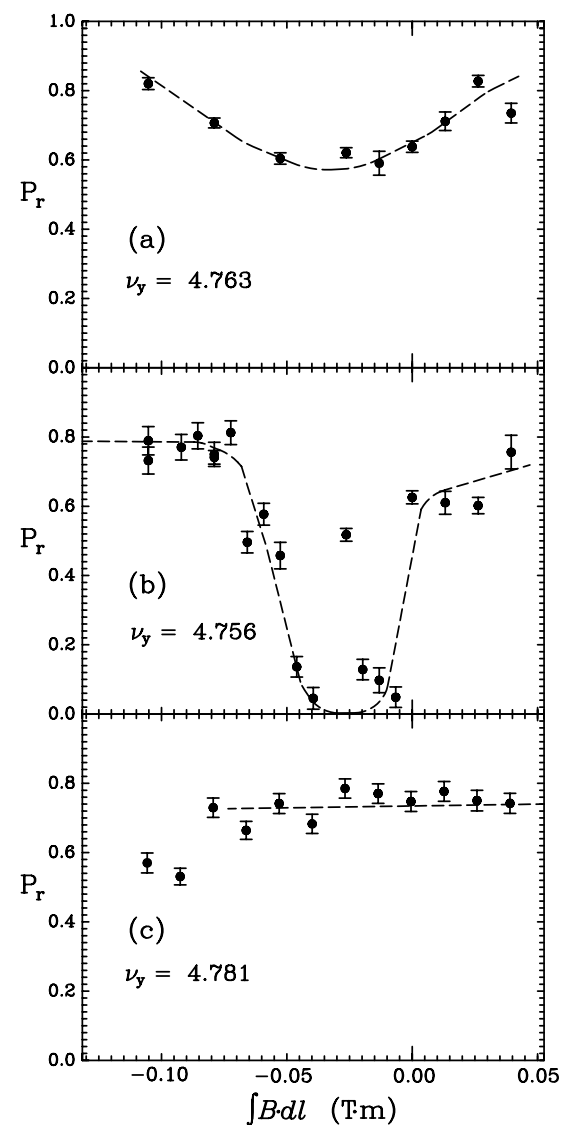

Figure 2: The measured radial polarization $P_{r}$ is plotted against the field integral in the correction solenoids (CSA and CSB) for different $\nu_{y}$ values. The dashed curves are hand-drawn lines to guide the eye.

one can effectively vary the spin tune at a rate of about $0.00036 \mathrm{~A}^{-1}$. We used this technique as a sensitive tool to study weak second-order snake depolarizing resonance.

When the vertical betatron tune was inadvertently set at 4.763 , we found the broad partial depolarization region, shown in Fig. 2a. The data suggested proximity to a second-order snake resonance because $\nu_{y}$ was near a quarter-integer. To test this hypothesis, we then changed $\nu_{y}$ to 4.756 , and observed the much stronger depolarization dip, shown in Fig. 2b. Finally, we moved $\nu_{y}$ away from the quarter-integer to 4.781. As expected, the beam polarization returned to its full value, as shown in Fig. 2c. Our future plans include further studies of higher-order snake resonances.

\section{SPIN-FLIPPING IN THE PRESENCE OF A FULL SIBERIAN SNAKE}

Spin-polarized beam experiments in storage rings such as the IUCF Cooler Ring, HERA [5], RHIC [6] and possibly Fermilab [7], require frequent reversals of the beam polarization direction to reduce the systematic errors in the measured asymmetry. An rf solenoid was used earlier to spin- flip a vertically polarized proton beam stored in a ring with no Siberian snake [8]. Since any very high energy ring will need full Siberian snakes to maintain the proton beam polarization, it is important to develop spin-flipping capability in the presence of a full snake. Using an rf solenoid, we made the first spin-flipping demonstration of a stored polarized proton beam with an efficiency of $91 \pm 1 \%$ [9]. We then tried to improve the spin-flipping efficiency by eliminating possible synchrotron sidebands and by using a weak rf dipole.

Even with a full Siberian snake in a ring, an rf magnetic field from either an rf solenoid or an rf dipole can induce an rf depolarizing resonance; this resonance is sometimes called a snake resonance, because it only exists at its frequency in the presence of a Siberian snake. Such resonances can be used to flip the spin direction of the ring's stored polarized protons.

The frequency $f_{r}$, at which an rf magnet can induce a depolarizing resonance, is given by

$$
f_{r}=f_{c}\left(k \pm \nu_{s}\right),
$$

where $f_{c}$ is the proton's circulation frequency, and $k$ is an integer. Slowly sweeping the rf magnet's frequency through $f_{r}$ can flip the spin. The Froissart-Stora equation [10] gives the ratio of $P_{f}$, the polarization after crossing the resonance, to the initial polarization $P_{i}$,

$$
P_{f}=P_{i}\left(2 \exp \left[\frac{-(\pi w)^{2}}{4 \Delta f / \Delta t}\right]-1\right),
$$

where $w$ is the resonance width in $\mathrm{Hz}$, and $\Delta f / \Delta t$ is the resonance crossing rate with $\Delta f$ being the frequency range during the ramp time $\Delta t$.

With a nearly full Siberian snake in the ring, the spin tune $\nu_{s}$ is very close, but is not exactly equal, to 0.5 . Thus, there

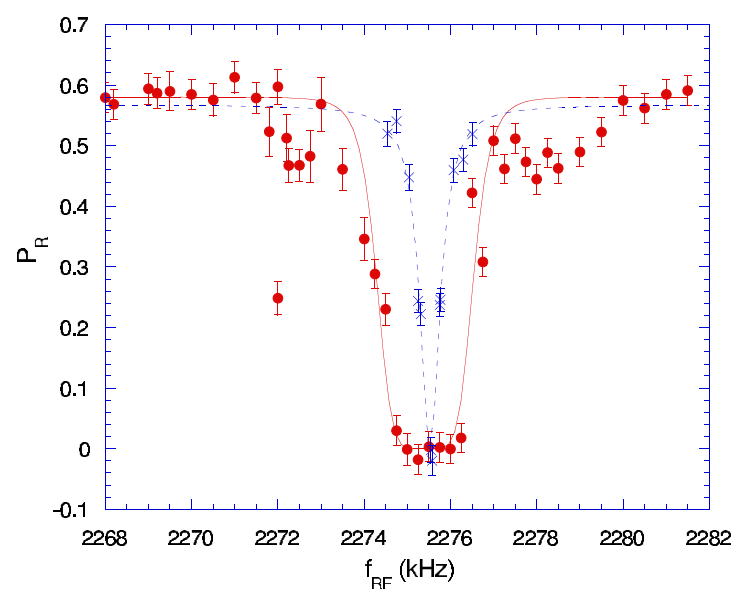

Figure 3: The measured radial proton polarization at 104.1 MeV is plotted against the frequency of the $\mathrm{rf}$ solenoid and of the rf dipole. The rf solenoid's data $(\bullet)$ are fitted with a third-order Lorentzian (solid curve). The rf dipole's data $(\times)$ are fitted with a first-order Lorentzian (dashed curve). 
should be two closely spaced rf depolarizing resonances around $1.5 f_{c}=2.2574 \mathrm{MHz}$. We excited these rf depolarizing resonances by operating the rf solenoid and the rf dipole near 2.25 MHz. The rf solenoid's amplitude was set at $6 \mathrm{kV}$, which corresponds to an $\int B \cdot d \ell=1.6 \mathrm{~T} \cdot \mathrm{mm}$; the single turn horizontal kicker was operated as an rf dipole at $23 \mathrm{~V}$ giving $\int B \cdot d \ell$ about $0.03 \mathrm{~T} \cdot \mathrm{mm}$. The measured radial polarization is plotted against the rf solenoid and rf dipole frequency in Fig. 3. For the rf solenoid's induced resonance, the resonance frequency and width were found to be $f_{1}=2,275,410 \pm 43 \mathrm{~Hz}$ and $w_{1}=2,270 \pm 90$ Hz. For the rf dipole's induced resonance, they were $f_{2}=2,275,530 \pm 10 \mathrm{~Hz}$ and $w_{2}=540 \pm 30 \mathrm{~Hz}$.

We then studied spin-flipping with a nearly full Siberian snake by crossing the rf induced snake resonance; we linearly ramped the frequency, of the rf solenoid or the rf dipole, through a frequency range $\Delta f$ which included the resonance frequency $f_{r}$ at various ramp times $\Delta t$. The radial beam polarization measured after each ramp is plotted in Fig. 4. Clearly the spin-flip efficiency is much better with the rf solenoid than with the rf dipole. By comparing the measured polarization of $58.2 \pm 2.1 \%$ at $\Delta t=0$ with the average polarization of $-20 \pm 2 \%$ at long dipole ramp times, we found a $30 \pm 4 \%$ dipole spin-flip efficiency.

To better estimate the solenoid's spin-flip efficiency, we measured the beam polarization after many spin-flips. We varied the number of spin-flips, while keeping the ramp time, frequency range and the rf voltage fixed; the data are shown in Fig. 5. We fit the data using the equation

$$
P=P_{i} \cdot \epsilon^{n},
$$

where $P_{i}$ is the initial polarization, $\epsilon$ is the spin-flip efficiency, and $n$ is the number of spin-flips. The best fit to the spin-flip efficiency is $97 \pm 1 \%$. We plan to study further this spin-flipping technique for both a nearly full and an exactly $100 \%$ Siberian snake.

\section{REFERENCES}

[1] A.D. Krisch et al., Phys. Rev. Lett. 63, 1137 (1989); J.E. Goodwin et al., Phys. Rev. Lett. 64, 2779 (1990).

[2] Ya.S. Derbenev and A.M. Kondratenko, Sov. Phys. Dokl. 20, 562 (1978).

[3] S.Y. Lee and S. Tepikian, Phys. Rev. Lett. 56, 1635 (1986).

[4] R.E. Pollock, Nucl. Instr. Meth. 300, 210 (1991).

[5] SPIN Collaboration and DESY Polarization Team, Acceleration of polarized protons to $820 \mathrm{GeV}$ at HERA, University of Michigan Report UM-HE 96-20 (1996).

[6] Y. Makdisi, Proc. 11th International Symposium on High Energy Spin Physics, Bloomington 1994, AIP Conf. Proc. 343, 75 (1995).

[7] SPIN Collaboration, Acceleration of polarized protons to $120 \mathrm{GeV}$ and $1 \mathrm{TeV}$ at Fermilab, University of Michigan Report UM-HE 95-09 (1995).

[8] D. D. Caussyn et al., Phys. Rev. Lett. 73, 2857 (1994).

[9] B.B. Blinov et al., Phys. Rev. Lett. 81, 2906 (1998).

[10] M. Froissart and R. Stora, Nucl. Instr. Meth. 7, 297 (1960).
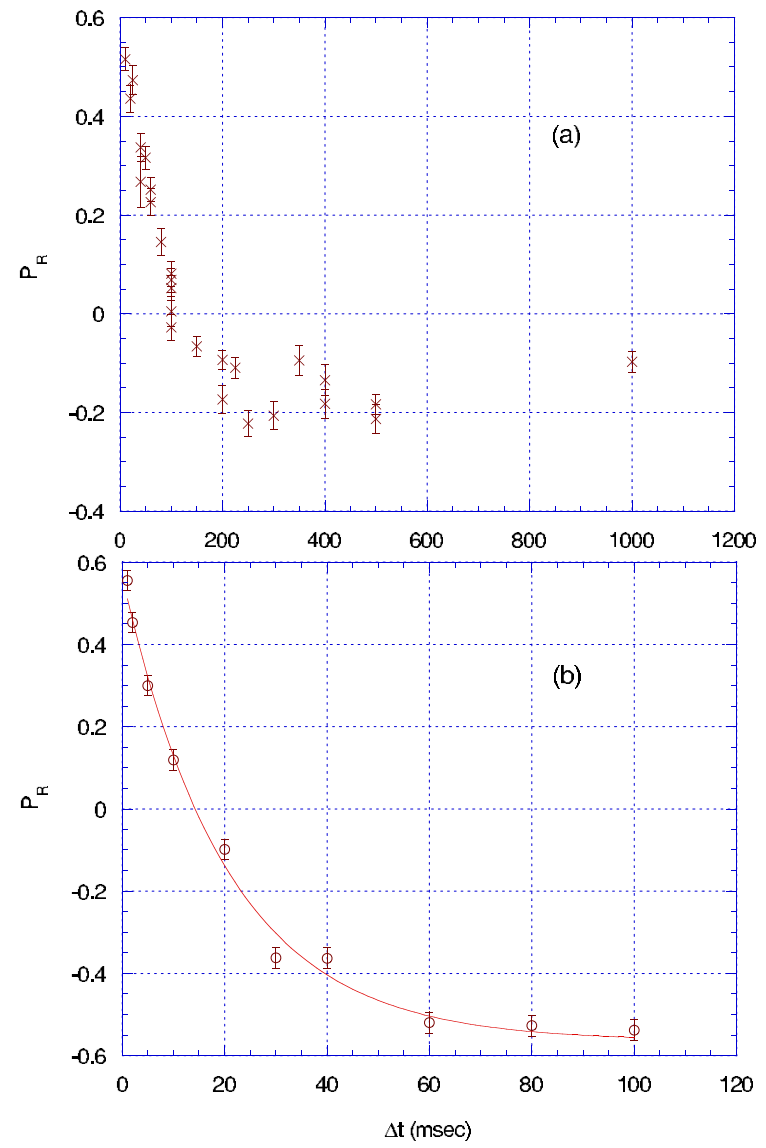

Figure 4: The measured radial proton polarization at 104.1 $\mathrm{MeV}$ is plotted against the ramp time $\Delta t$ of the rf dipole (a) and the rf solenoid (b). The frequency ranges $\Delta f$ were $3 \mathrm{kHz}$ for the rf dipole, and $12 \mathrm{kHz}$ for the $\mathrm{rf}$ solenoid. The curve is a fit to the data using Eq. (5).

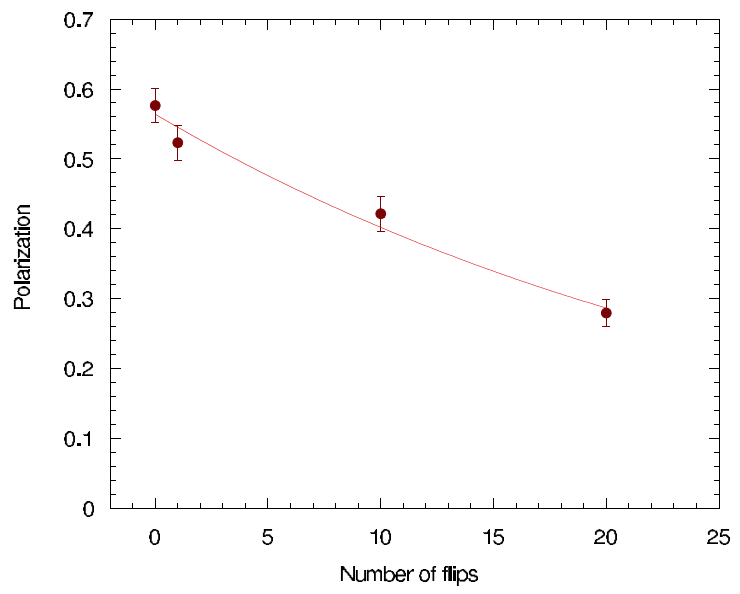

Figure 5: The measured radial proton polarization at 104.1 $\mathrm{MeV}$ is plotted against the number of spin-flips. The rf solenoid's ramp time $\Delta t$ is $60 \mathrm{msec}$, and its frequency range $\Delta f$ is $3.5 \mathrm{kHz}$. The curve is a fit using Eq.(6). 